\title{
Tim B. Müller, Adam Tooze (dir.), Normalität und Fragilität. Demokratie nach dem Ersten Weltkrieg
}

\section{Marcel Tambarin}

\section{OpenEdition}

\section{Journals}

Édition électronique

URL : http://journals.openedition.org/ifha/8876

DOI : $10.4000 /$ ifha. 8876

ISSN : 2198-8943

Éditeur

IFRA - Institut franco-allemand (sciences historiques et sociales)

Référence électronique

Marcel Tambarin, «Tim B. Müller, Adam Tooze (dir.), Normalität und Fragilität. Demokratie nach dem Ersten Weltkrieg », Revue de l'IFHA [En ligne], Date de recension, mis en ligne le 02 novembre 2017, consulté le 24 septembre 2020. URL : http://journals.openedition.org/ifha/8876 ; DOI : https://doi.org/ $10.4000 /$ ifha. 8876

Ce document a été généré automatiquement le 24 septembre 2020.

(C)IFHA 


\section{Tim B. Müller, Adam Tooze (dir.), Normalität und Fragilität. Demokratie nach dem Ersten Weltkrieg}

Marcel Tambarin

\section{RÉFÉRENCE}

Tim B. Müller, Adam Tooze (dir.), Normalität und Fragilität. Demokratie nach dem Ersten Weltkrieg, Hamburg: Hamburger Edition, 2015, 518 p., $35 €$ 
Lorsqu'en 1956 Fritz René Allemann affirme que «Bonn n'est pas Weimar» pour mieux souligner les aspects positifs de la nouvelle démocratie ouestallemande, il conforte en même temps l'image négative de la République de Weimar, dont l'échec sert le plus souvent de repoussoir dans le récit national allemand. C'est précisément cette interprétation orientée de Weimar, et plus généralement des expériences démocratiques de l'entre-deux-guerres en Europe, que prétend revisiter et remettre en question ce volume édité par Tim B. Müller, du Hamburger Institut für Sozialforschung (HIS), et par Adam Tooze, directeur de l'European Institute de l'université Columbia de New York.

Définissant explicitement leur sujet comme le making of democracy (p.9), und Fragilität Demokratie mach dem Ersten Weltkrieg

Hamburger IEdition T. Müller et A. Tooze ne considèrent pas la démocratie comme un idéal-type, mais comme un processus, suivant le mot de Pierre Rosanvallon constatant que la démocratie n'a pas seulement une histoire, mais qu'elle est une histoire. Voulant échapper au piège de «l'obsession embryogénétique » (p. 14), cette approche exclut toute lecture téléologique de l'entre-deux-guerres, toute interprétation de la République de Weimar en particulier comme prodrome de la catastrophe de la Seconde Guerre mondiale. Elle propose tout au contraire de considérer la période qui commence avec la Première Guerre mondiale comme une " phase constitutive de la démocratie moderne » (p. 23) en émancipant l'interprétation de l'entre-deux-guerres de la période qui l'a suivie. Il s'agit aussi de dépasser le cadre narratif national en faisant ressortir les aspects sinon universels, $d u$ moins transnationaux du phénomène démocratique.

L'ouvrage est divisé en deux parties comprenant respectivement 7 et 13 contributions remontant à un colloque organisé au Hamburger Institut für Sozialforschung. Elles sont précédées d'un long chapitre introductif dans lequel les deux coéditeurs exposent leur approche et leur démarche, qui s'inscrit dans le cadre de l'histoire culturelle des concepts politiques, mais non toutefois dans celui d'une "simple " histoire des idées dans la mesure où les auteurs revendiquent dans leurs réflexions une place centrale pour l'économie et la politique économique.

La première partie ( Constellations, continuités et convergences ») aborde les grands thèmes de la période dans une perspective transnationale: le processus de dynamisation démocratique enclenché à partir de 1914 (A. Tooze), les techniques électorales et la constitution de l'électorat moderne en Europe et en Amérique du Nord (Hedwig Richter), les défis posés aux partis dans le nouveau contexte électoral (Benjamin Schröder), les perspectives et les limites de l'engagement des femmes dans la société civile (Laura Beers), les rapports entre corporatisme et démocratie (Andrea 
Rehling), ceux entre démocratie parlementaire et capitalisme (Philipp Müller) et enfin les débats autour de la question du leadership en démocratie (Moritz Föllmer).

La seconde et plus importante partie de l'ouvrage («Contextes nationaux, conflits et contingences») met en œuvre le but affiché dans l'introduction, à savoir montrer et analyser les formes concrètes de réalisation de la démocratie dans l'entre-deux-guerres dans différents contextes nationaux. Bien que portant entièrement sur l'Europe occidentale, à l'exception du chapitre consacré au New Deal (Jason Scott Smith), et bien que l'accent soit mis sur la République de Weimar dans les trois contributions de T. Müller, Philipp Nielsen et Stefanie Middendorf, le choix est assez large pour couvrir une grande diversité de cas: la Grande-Bretagne (dans les contributions d'Helen MacCarthy et de Ben Jackson), la France (Jessica Wardhaugh), la Suède (Urban Lundberg), le Danemark (Jeppe Nevers), la Finlande (Johanna Rainio-Niemi), les PaysBas (Elisabeth Dieterman), la Tchécoslovaquie (Andrea Orzoff) et l'Espagne (Till Kössler).

L'ensemble des deux parties constitue un vaste panorama des expérimentations démocratiques et de leurs évolutions nationales dans l'entre-deux-guerres. Les questions centrales posées dans l'introduction, qui portent sur « l'émergence de la démocratie comme valeur en soi, l'acceptation de la démocratie, la variété de ses formes d'expression dans la politique et dans la vie quotidienne, la formation d'un horizon d'attente démocratique général » (p. 19), y trouvent des réponses instructives même si l'on peut regretter par exemple que le rôle et l'influence de l'industrie, dans l'Allemagne de Weimar en particulier, n'aient pas davantage retenu l'attention. Mais l'étude du cas français illustre l'intérêt d'une démarche comparative questionnant les catégorisations convenues : J. Wardhaugh montre ainsi, en croisant archives de partis, articles de presse et rapports de police, que bien des oppositions relevaient davantage de la rhétorique politique qu'elles n'étaient inscrites dans la réalité sociale et que certaines revendications du Front populaire par exemple se trouvaient partagées jusque par les Croix de feu. En d'autres termes, l'opposition classique droite-gauche ne recoupait pas forcément l'opposition entre opposants à et partisans de la démocratie, ce qui signifie aussi qu'une approche dichotomique de cette période contribue davantage à en brouiller l'interprétation qu'à l'éclairer.

Le titre du volume traduit du reste parfaitement cette ambivalence du processus démocratique de l'entre-deux-guerres, c'est-à-dire aussi bien ses potentialités inégalement exploitées que ses failles cristallisées dans l'échec de Weimar. La présente réévaluation, sinon réhabilitation, en propose néanmoins une nouvelle « lecture modérément optimiste » et relance à tout le moins la discussion sur cette période historique qui a vu dans de nombreuses sociétés la démocratie s'instaurer comme horizon des possibles puis comme horizon d'attente.

\section{INDEX}

Index chronologique : Époque contemporaine

Thèmes : Histoire des États et des pouvoirs, Histoire des idées 


\section{AUTEURS}

\section{MARCEL TAMBARIN}

Université de Bourgogne Franche-Comté (Dijon) - ILCEA4/CERAAC (EA 7356) 